\title{
PAPER \\ Study of Dispersion of Lightning Whistlers Observed by Akebono Satellite in the Earth's Plasmasphere
}

\author{
I Putu Agung BAYUPATI ${ }^{\dagger}$, Nonmember, Yoshiya KASAHARA ${ }^{\dagger \dagger a)}$, and Yoshitaka GOTO ${ }^{\dagger \dagger \dagger}$, Members
}

\begin{abstract}
SUMMARY When the Akebono (EXOS-D) satellite passed through the plasmasphere, a series of lightning whistlers was observed by its ana$\log$ wideband receiver (WBA). Recently, we developed an intelligent algorithm to detect lightning whistlers from WBA data. In this study, we analyzed two typical events representing the clear dispersion characteristics of lightning whistlers along the trajectory of Akebono. The event on March 20, 1991 was observed at latitudes ranging from $47.83^{\circ}\left(47,83^{\circ} \mathrm{N}\right)$ to $-11.09^{\circ}\left(11.09^{\circ} \mathrm{S}\right)$ and altitudes between $\sim 2232$ and $\sim 7537 \mathrm{~km}$. The other event on July 12,1989 was observed at latitudes from $34.94^{\circ}\left(34.94^{\circ} \mathrm{N}\right)$ and $-41.89^{\circ}\left(41.89^{\circ} \mathrm{S}\right)$ and altitudes $\sim 1420-\sim 7911 \mathrm{~km}$. These events show systematic trends; hence, we can easily determine whether the wave packets of lightning whistlers originated from lightning strikes in the northern or the southern hemispheres. Finally, we approximated the path lengths of these lightning whistlers from the source to the observation points along the Akebono trajectory. In the calculations, we assumed the dipole model as a geomagnetic field and two types of simple electron density profiles in which the electron density is inversely proportional to the cube of the geocentric distance. By scrutinizing the dipole model we propose some models of dispersion characteristic that proportional to the electron density. It was demonstrated that the dispersion D theoretically agrees with observed dispersion trend. While our current estimation is simple, it shows that the difference between our estimation and observation data is mainly due to the electron density profile. Furthermore, the dispersion analysis of lightning whistlers is a useful technique for reconstructing the electron density profile in the Earth's plasmasphere.

key words: lightning, whistler, dispersion, path length, plasmasphere, electron density
\end{abstract}

\section{Introduction}

Akebono (EXOS-D) is a Japanese scientific spacecraft that has been observing the Earth's plasmasphere since 1989. A wide band analyzer (WBA) is a subsystem of the VLF instruments onboard Akebono, and it measures one component of the electric or magnetic waveform below $15 \mathrm{kHz}$ [1]. The measured data is sent to the ground using analog telemetry. While orbiting the Earth, the WBA detected large amounts of analog waveforms [2], and one of the most frequently observed wave signals was "lightning whistlers," which are characterized by their dispersed spectra. Lightning whistlers were discovered in the 19th century as elec-

\section{Manuscript received April 3, 2012}

Manuscript revised July 12, 2012.

$\dagger$ The author is with the Graduate School of Natural Science and Technology, Kanazawa University, Kanazawa-shi, 920-1192 Japan.

${ }^{\dagger}$ The author is with Information Media Center, Kanazawa University, Kanazawa-shi, 920-1192 Japan.

$+{ }^{\dagger \dagger}$ The author is with Faculty of Electrical and Computer Engineering, Kanazawa University, Kanazawa-shi, 920-1192 Japan.

a)E-mail: kasahara@is.t.kanazawa-u.ac.jp

DOI: 10.1587/transcom.E95.B.3472 tromagnetic waves that originate from lightning strikes and propagate through the Earth's plasmasphere at audio frequencies [3]-[5]. In the plasmasphere, a lightning strike is converted into a whistling tone; therefore, this type of wave is called a "whistler mode wave" [3]. It is well known that whistler mode waves tend to propagate along magnetic field lines from the southern to the northern hemisphere, or vice-versa [5], [6], and the propagation velocity of whistler mode waves becomes slower in the lower frequency range. A lightning whistler is originally an impulse signal, but the spectrum is characterized by a discrete tone that decreases in frequency with time. This is caused by the velocity difference between higher and lower frequency components as the whistler propagates through the plasmasphere [7], [8]. The dispersive spectrum property (decrease in frequency with time) is generally defined as "dispersion," which is an important indicator of the length of the propagation path and the plasma environment along the propagation path.

In the satellite era, lightning whistlers are commonly observed from spacecraft because they can be more frequently observed from space than from the ground [3], [9]. Such lightning whistlers are categorized as nonducted whistlers, and their energy can propagate thousands of kilometers from the source of the lightning strike in the plasmasphere [9], [10]. Akebono has operated successfully for more than 23 years since its launch, and enormous wealth of waveform data, including that of lightning whistlers, has been captured by the WBA.

This paper analyzes the "dispersion" of lightning whistlers along the trajectories of Akebono. In particular, we focus on the dispersion trend along trajectories that provide useful information about the source of lightning whistlers and the plasma environment, such as the electron density along the propagation path. It is well known that the global plasma density in the Earth's plasmasphere drastically varies with local time, season and solar activity. However, it is difficult to measure the global density profile directly because spacecraft observations can only provide in situ electron density along the trajectory and multisatellites are necessary in order to cover over large region [11]. On the other hand, Goto et al. [12] proposed an intelligent method to derive global electron density using propagation characteristics of Omega signals. Unfortunately transmission of Omega signal was already terminated, but if we could apply the same method to the propagation characteristics of lightning whistlers instead of Omega, it will be more cheap and effective tool for its investigation. Therefore, we 


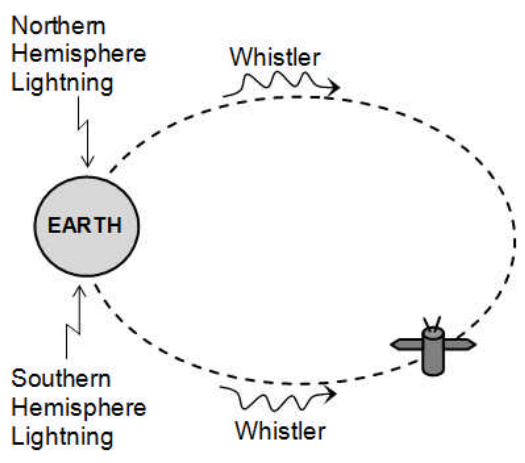

Fig. 1 Propagation of lightning whistler along magnetic field line in the plasmasphere.

study trends of "dispersion" which reflect the propagation characteristics of the lightning whistlers and compare them with those which are derived theoretically. The analysis shows that the source of whistler waves will easily determine whether whistlers generated by lightning strikes are guided along the magnetic field lines from the southern or the northern hemispheres, as shown in Fig. 1 [13]. In addition, studying the dispersion trend helps to determine the electron density profile in the Earth's plasmasphere, because the propagation velocity of whistler mode waves depends on the intensity of the ambient magnetic field and the electron density around the Earth [6], [14]. Therefore, it is important to extrapolate the electron density profile of the entire plasmasphere by examining the dispersion trend along the spacecraft trajectory. For these reasons, we analyze the trend of the whistler dispersion in relation to parameters, particularly electron density profile in the plasmasphere. Finally, we discuss whether the observed dispersion trends agree with the ones theoretically derived, and we show the feasibility of modeling the electron density profile using numerous datasets obtained by the WBA onboard Akebono.

\section{Observation}

In this study, we utilize the WBA waveform data observed by the WBA onboard Akebono. The WBA data were originally recorded and stored in digital audio tapes (DATs). In the DATs, the analog time code signal, which indicates universal time and date, is recorded on the left channel, while the measured waveform signal is recorded on the right channel [1]. To analyze the data, it is necessary to playback the tape and converts it to waveform data represented by the waveform audio file format (WAV), digitizing by an A/D converter with a sampling frequency of $48 \mathrm{kHz}$. To generate a plot showing the variation of frequency with time, we performed a Finite Fourier Transform (FFT) every $0.05 \mathrm{~s}$. After FFT processing, we calibrated the spectrum based on the housekeeping data related to the autogain controller implemented in the WBA. We also eliminated spike noises that contaminated the waveform data. In the noise reduction process, we divided the $50 \mathrm{~Hz}-15 \mathrm{kHz}$ band into 20 frequency ranges, then averaged the intensity of each frequency band,

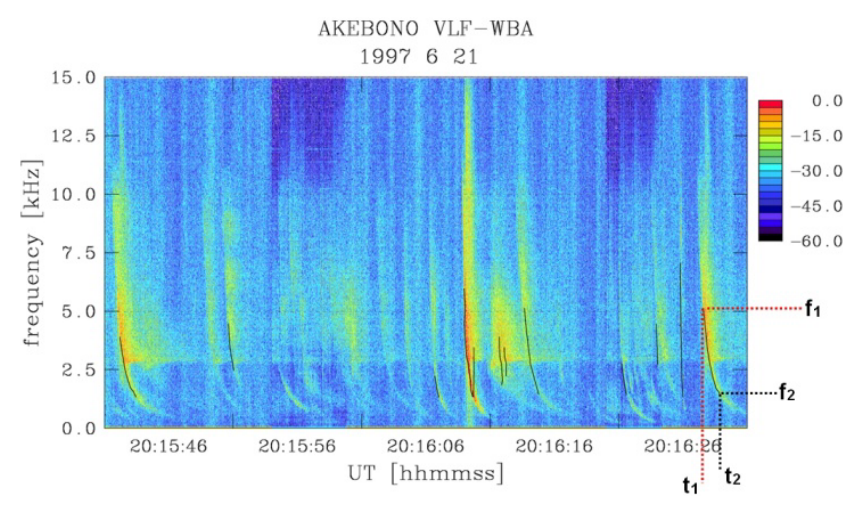

Fig. 2 Detected lightning whistlers.

and subtracted neighboring frequency points. For the next step, we defined a threshold level for the detection of significant signals. If the intensity of differential data is larger than the threshold, it is considered to be a potential lightning whistler candidate. It is theoretically well known that Eckersley's law [15] provides a simple relationship that defines the arrival time $t$ of a lightning whistler at a frequency $f$ as follows

$$
t=D / \sqrt{f}+t_{0}
$$

where $D$ is a constant called dispersion, $t$ is the arrival time at frequency $f$, and $t_{0}$ is the time when the lightning strikes. Then, we applied an automatic algorithm to detect straight lines in a diagram in which the spectrogram is converted into $t$ in the horizontal axis and $1 / \sqrt{f}$ in the vertical axis. An example of lightning whistlers detected by this process is shown in Fig. 2, which is well marked by thick curves. The figure shows one of them symbolized by $t_{1}, f_{1}, t_{2}$ and $f_{2}$, where $t_{1}$ and $t_{2}$ are the arrival times of the detected lightning whistler at frequencies of $f_{1}$ and $f_{2}$, respectively.

Finally, we compute the dispersion $D$ using the following equation

$$
D=\frac{t_{2}-t_{1}}{\frac{1}{\sqrt{f_{2}}}-\frac{1}{\sqrt{f_{1}}}}
$$

By using Eq. (2), all dispersions of the detected lightning whistlers can be automatically enabled.

\section{Dispersion Analysis}

As mentioned previously, the dispersion measured along the trajectory of a spacecraft can provide useful information for determining the plasma parameters in the Earth's plasmasphere. Here, we will investigate the path length further by analyzing the dispersion. The criterion to select these events is the clarity of the dispersion trend of a lightning whistler, which has a systematic trend. Theoretically, a slope trend is tangible evidence that the whistler has a strong correlation with the origins of lightning strikes for the observed lightning whistler data. This indicates that the source of the observed lightning whistler data can be determined from the 
trajectory of Akebono.

In this section, we first theoretically describe the relationship between the dispersion and the propagation path length to show that the dispersion trend of lightning whistlers is useful for determining the plasma environment inside the Earth's plasmasphere. As stated previously, the dispersion depends on several parameters, such as the intensity of the ambient magnetic field, the electron density, and the path length along the propagation paths. The path length between the source point of lightning strikes and the observation point in the Earth's plasmasphere affects the dispersion scale of lightning whistlers [9], [16]. Short path lengths lead to minimum dispersion, and vice versa [9], [16]. Furthermore, to estimate the path length, we can utilize a simple method by assuming that the structure of the Earth's magnetic field is approximated by a simple magnetic dipole model [17], [18]. By adopting the approximation, we can briefly calculate the path length of the lightning whistler to demonstrate why the dispersion changes along the trajectory of Akebono in such a manner.

Using parameters such as the altitude and the latitude of the Akebono trajectory, it is possible to calculate the path length of each lightning whistler detected along the Akebono trajectories, assuming that each signal propagates along the magnetic field line of the Earth from the ground to the observation point. Note that whistler mode wave does not propagate strictly along the magnetic field line in actual, but we assume here that the wave propagates along the magnetic field line with its wave normal angle parallel to the ambient magnetic field for simple calculation purposes. Then we analyze the approximated equation from a model of a dipole magnetic field line. Figure 3 shows a schematic picture of the magnetic field line at a given point in the plasmasphere. This figure shows the relationship between the geocentric distance $r$ and the magnetic colatitude $\theta$ of any point on a field line [18]. In this case, let $\beta$ be the angle between the magnetic field line and the latitude line. $B_{\theta}$ and $B_{r}$ represent the horizontal component and the vertical component of the magnetic field, respectively. The figure also shows the X-axis, which corresponds to the south geomag-

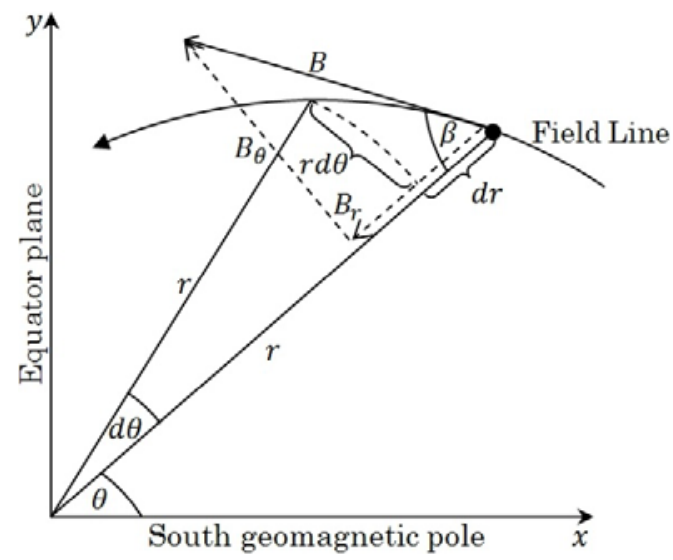

Fig. 3 Relationship between the radius vector and latitude of a field line. netic pole of the Earth, while the y-axis corresponds to the equatorial plane of the Earth.

From the previous figure, the following equations can be derived:

$$
\begin{aligned}
& B_{\theta}=B_{0} \frac{1}{r^{3}} \sin \theta \\
& B_{r}=2 B_{0} \frac{1}{r^{3}} \cos \theta \\
& \frac{r d \theta}{d r} \approx \tan \beta=\frac{B_{\theta}}{B_{r}}=\frac{1}{2} \frac{\sin \theta}{\cos \theta}=\frac{1}{2} \tan \theta
\end{aligned}
$$

where $B_{0}$ is the equatorial surface magnetic field intensity of the Earth. After deriving the equations, we obtain the solution

$$
\cos \beta=\frac{2 \cos \theta}{\sqrt{1+3 \cos ^{2} \theta}}
$$

To calculate the path length, we substitute $\left(90+\theta^{\prime}\right)$ for $\theta$, where $\theta^{\prime}$ is defined as the magnetic latitude. In accordance with Fig. 4, we obtain the following equation

$$
d r=\cos \beta d s=-\frac{2 \sin \theta^{\prime}}{\sqrt{1+3 \sin ^{2} \theta^{\prime}}} d s
$$

where $d s$ is an element of the path length along a magnetic field line. Then, the path length $S$ is calculated using the following integration:

$$
S=\int d s=-\int_{\theta_{0}^{\prime}}^{\theta_{1}^{\prime}} \frac{\sqrt{1+3 \sin ^{2} \theta^{\prime}}}{2 \sin \theta^{\prime}} d r
$$

We then consider a shell parameter $L$ that corresponds to the distance, which is an intersection of the field line and the equatorial plane, and is given in units of Earth radii. According to [18], [19], $d r$ is given by the following form

$$
d r=-R_{E} \cdot L \cdot 2 \cos \theta^{\prime} \sin \theta^{\prime} d \theta^{\prime}
$$

where $R_{E}$ is the radius of the Earth, which is approximately $6371 \mathrm{~km}$. Under these circumstances, the following equation is derived.

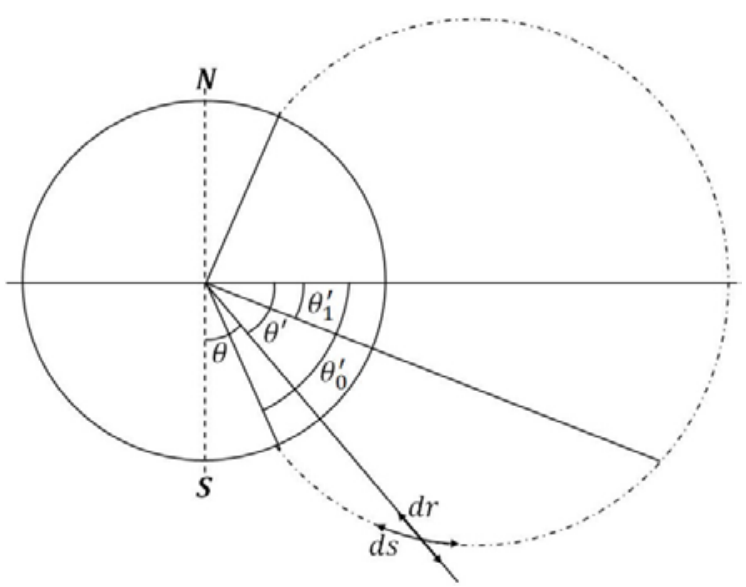

Fig. 4 Dipole field line approximation of plasmasphere field line. 


$$
S=\int_{\theta_{0}^{\prime}}^{\theta_{1}^{\prime}} \frac{\sqrt{1+3 \sin ^{2} \theta^{\prime}}}{2 \sin \theta^{\prime}} R_{E} \cdot L \cdot 2 \cos \theta^{\prime} \sin \theta^{\prime} d \theta^{\prime}
$$

Let $x=\sin \theta^{\prime}$; thus, $d x=\cos \theta^{\prime} d \theta^{\prime}$. We now acquire the last equation to calculate the path length between the source point of the whistler wave and the spacecraft along the ambient magnetic field line.

$$
S=R_{E} \cdot L \int_{x_{0}}^{x_{1}} \sqrt{1+3 x^{2}} d x
$$

By using the equation, we can estimate each path length of the detected lightning whistler from the source point.

When an element of the path length is well defined, we can derive the lightning whistler dispersion along each propagation path theoretically. The propagation delay time $t_{d}$, which is given by $\left(t-t_{0}\right)$ in Eq. (1), is expressed by using the following equation when the lightning whistler propagates along the ambient magnetic field line with its wave normal parallel to the magnetic field [20]

$$
t_{d}=\frac{1}{c} \int \mu_{g} d s=\frac{1}{2 c} \int \frac{f_{p} f_{c}}{f^{1 / 2}\left(f_{c}-f\right)^{3 / 2}} d s
$$

where $c, \mu_{g}, f, f_{p}$, and $f_{c}$ are the speed of light, group refractive index, wave frequency, plasma frequency, and electron gyrofrequency, respectively. Based on Eqs. (1) and (12), we can obtain the following equation

$$
D=\frac{1}{2 c} \int \frac{f_{p} f_{c}}{\left(f_{c}-f\right)^{3 / 2}} d s
$$

When the wave frequency is significantly less than the local electron gyrofrequency, i.e., $f_{c} \gg f$, Eq. (13) is approximately described as follows

$$
D=\frac{1}{2 c} \int \frac{f_{p}}{\sqrt{f_{c}}} d s
$$

It is well known that the local electron plasma frequency is proportional to $N^{1 / 2}$, where $N$ is the number density of electrons, and the local electron gyrofrequency is proportional to the geomagnetic field strength $B$ [20]. As was proposed by Carpenter and Smith [21], if the electron density in the plasmasphere is inversely proportional to the cube of the geocentric distance as shown by Eq. (15) (hereafter we define this electron density profile as "Model 1"), we can obtain the following equations

$$
\begin{aligned}
& N_{1}=\frac{N_{0}}{r^{3}} \\
& f_{p} \propto \sqrt{N_{1}} \propto \frac{1}{\sqrt{r^{3}}} \\
& f_{c} \propto B=B_{0} \frac{\sqrt{1+3 \sin ^{2} \theta^{\prime}}}{r^{3}}
\end{aligned}
$$

where $N_{0}$ are the electron density at upper ionosphere of the Earth. Combining Eqs. (14), (16), and (17), we obtain

$$
D_{1} \propto \int \frac{1}{\left(1+3 \sin ^{2} \theta^{\prime}\right)^{1 / 4}} d s
$$

where $D_{1}$ is the dispersion at the observation point along the spacecraft trajectory when the electron density in the plasmasphere is represented by Eq. (15) ("Model 1"). By replacing in Eq. (18) by Eq. (7) and replacing $d r$ by Eq. (9), we can derive the dispersion using the following equation

$D_{1} \propto \int \frac{\left(1+3 \sin ^{2} \theta^{\prime}\right)^{1 / 4}}{2 \sin \theta^{\prime}} d r=\int\left(1+3 \sin ^{2} \theta^{\prime}\right)^{1 / 4} \cos \theta^{\prime} d \theta^{\prime}$

By letting $x=\sin \theta^{\prime}$, we get the equation to calculate the dispersion

$$
D_{1} \propto \int\left(1+3 x^{2}\right)^{1 / 4} d x
$$

On the other hand, if the electron density in the plasmasphere is, for example, given by Eq. (21) (hereafter we define this electron density profile as "Model 2"), which is known as gyrofrequency model [22], assuming that electron density is larger in the lower latitude region than the higher latitude region

$$
N_{2}=\frac{N_{0}}{r^{3} \sqrt{1+3 \sin ^{2} \theta^{\prime}}}
$$

we can obtain the dispersion $D_{2}$ by combining Eqs. (14), (17) and (21) as follows

$$
D_{2} \propto \int \frac{1}{\sqrt{1+3 \sin ^{2} \theta^{\prime}}} d s=\int \cos \theta^{\prime} d \theta^{\prime}
$$

After the dispersions $D_{1}$ and $D_{2}$ are derived by assuming the electron density profile models, we introduce the typical observation events in which lightning whistlers are continuously observed along the trajectories of Akebono. We show the dispersion of these lightning whistlers has clear trend along the trajectory and we demonstrate that we can roughly estimate the electron density profile in the plasmasphere by comparing the observed dispersion trends with those theoretically derived.

\section{Result and Discussion}

Figure 5 shows a train of lightning whistlers that lasted from 19:13 to 19:51 UT on March 20, 1991. Such lightning whistler trains occur because of the abundance of waves from the lightning strike detected by Akebono. The figure shows the dispersion trends of lightning whistlers, which descend when the time increases. The number of lightning whistlers recorded during the time is 564 , and the dispersions vary between $\sim 12.81$ and $\sim 4.194 \mathrm{~Hz}^{1 / 2}$ s. In accordance with the Akebono trajectory orbit, the lightning whistlers in the figure are detected along the Akebono trajectory between latitudes $47.83^{\circ}\left(47,83^{\circ} \mathrm{N}\right)$ and $-11.09^{\circ}$ $\left(11.09^{\circ} \mathrm{S}\right)$ and altitudes from $\sim 2232$ to $\sim 7537 \mathrm{~km}$, as represented in Fig. 6. We utilized Eq. (11) to estimate the path lengths from the source points to the detected lightning whistlers assuming that the source points are located in the southern hemisphere. For the event on March 20, 1991 from 19:13 to 19:51 UT, the results of path lengths 


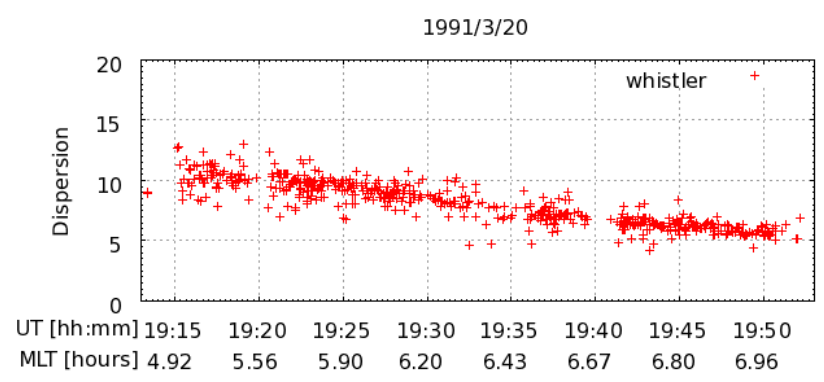

Fig. 5 Dispersion of lightning whistler observed on March 20, 1991.

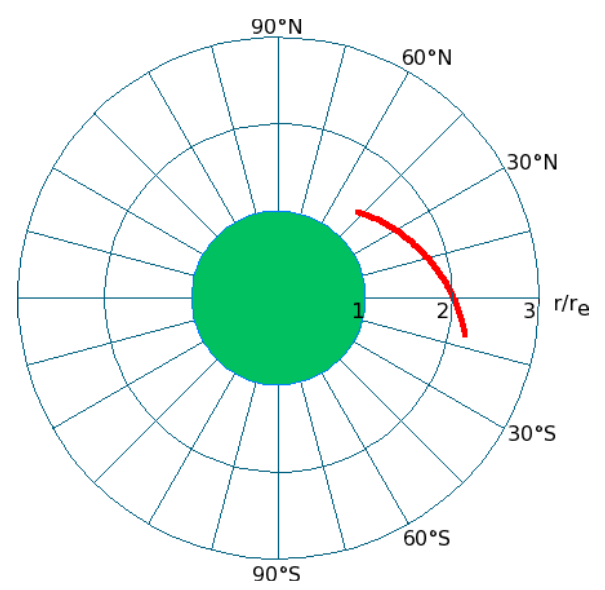

Fig. 6 Altitude and latitude of Akebono on March 20, 1991.

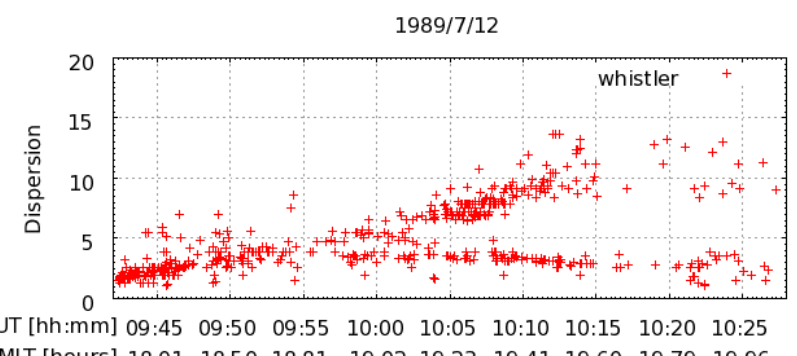

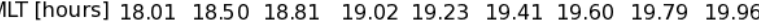

Fig. 7 Dispersion of lightning whistler observed on July 12, 1989.

decreased gradually from $\sim 49977$ to $\sim 10498 \mathrm{~km}$. Because a decrease in the path lengths over time will decrease the dispersion scale, this fact suggests that the waves of lightning whistler strikes propagate from the southern to the northern hemisphere.

Figure 7 shows a train of lightning whistlers that occurred from 09:42 to 10:26 UT on July 12, 1989. The number of lightning whistlers that were detected during that period of time was 542 . The dispersions vary between $\sim 1.11$ and $\sim 13.66 \mathrm{~Hz}^{1 / 2} \mathrm{~s}$. They were detected along the Akebono trajectory between latitudes $34.94^{\circ}\left(34.94^{\circ} \mathrm{N}\right)$ and $-41.89^{\circ}$ $\left(41.89^{\circ} \mathrm{S}\right)$ and altitudes from $\sim 1420$ to $\sim 7911 \mathrm{~km}$, as represented in Fig. 8.

Two dispersion trends can be observed coincidentally in Fig. 7. The phenomenon occasionally occurred because lightning whistler sources originated from the southern and

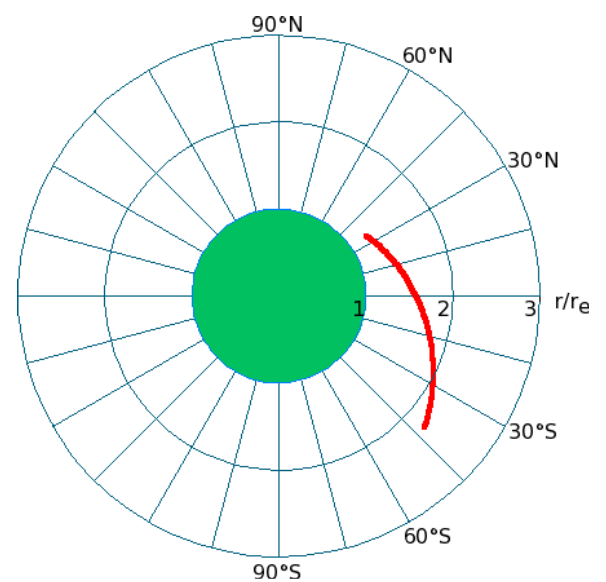

Fig. 8 Altitude and latitude of Akebono on July 12, 1989.

the northern hemispheres and were detected simultaneously. As shown in Fig. 7, one has an ascending trend, while the other has a slightly descending trend. By using the altitude and latitude of the Akebono orbit, as shown in Fig. 8, we calculated the path lengths of lightning whistler waves that originated from both the northern and the southern hemispheres. Between 09:42 and 10:26 UT, the path lengths of lightning whistler waves originating from the northern hemisphere toward Akebono became larger from $~ 1678$ to $\sim 65849 \mathrm{~km}$ with increasing time. One of the dispersion trends in Fig. 7 shows that the dispersion scale average was approximately $\sim 2 \mathrm{~Hz}^{1 / 2}$ s at 09:42 $\mathrm{UT}$ and gradually increased, becoming $\sim 12 \mathrm{~Hz}^{1 / 2} \mathrm{~s}$ at 10:26 UT.

In contrast, the path lengths of lightning whistler calculated from the source points in the southern hemisphere toward Akebono was $\sim 21210 \mathrm{~km}$ at 09:42 UT while it was $\sim 8577 \mathrm{~km}$ at 10:26 UT. Another dispersion trend in Fig. 7 shows that the dispersion scale becomes smaller from an average of $\sim 6 \mathrm{~Hz}^{1 / 2} \mathrm{~s}$ to $\sim 2 \mathrm{~Hz}^{1 / 2} \mathrm{~s}$ from 09:42 to 10:26 UT.

It can be conclusively stated that the ascending dispersion trend in Fig. 7 shows waves of lightning whistler strikes propagate from the northern to the southern hemisphere. The other descending trend shows the waves of lightning whistler strikes propagate from the southern to the northern hemisphere. The VLF instruments onboard Akebono has a subsystem named PFX to determine wave normal direction and Poynting vector [1], but the measuring frequency being set for PFX on this date was too high to detect lightning whistlers and it was unfortunately impossible to determine the propagation direction of the whistlers.

Next we investigate the relationship between these dispersion trends and the electron density profiles in the plasmasphere defined as Model 1 and Model 2. Figure 9 shows the dispersion trend observed on March 20, 1991 and the line of dispersion $D_{1}$ which was theoretically calculated by using Eq. (20). Note that $D_{1}$ is calculated from the right side of Eq. (20), and thus the scale is different from the observed dispersion. As known previously, the sources of lightning whistlers in this event originate from the southern hemisphere. Therefore the lightning whistlers propa- 


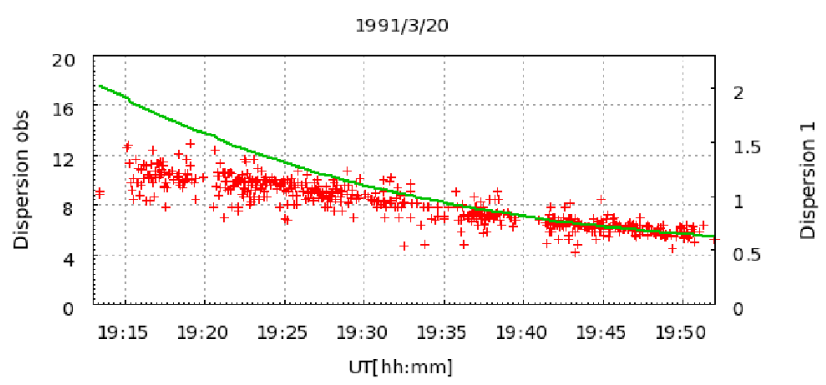

Fig. 9 Relationship between dispersion observed on March 20, 1991 (red symbols) and the one theoretically calculated by Model 1 (green line).

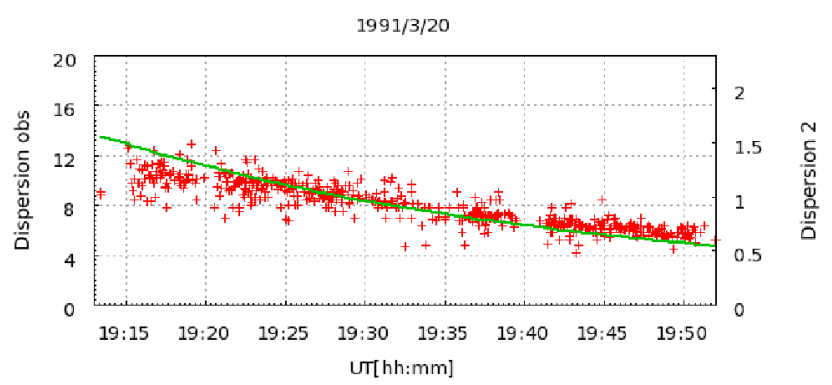

Fig. 10 Relationship between dispersion observed on March 20, 1991 (red symbols) and the one theoretically calculated by Model 2 (green line).

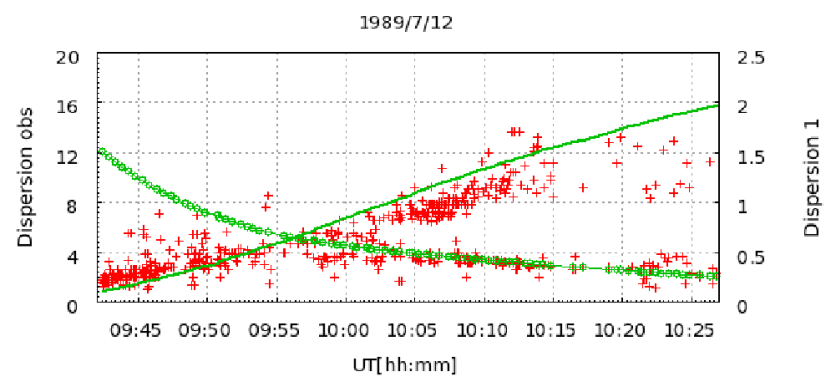

Fig. 11 Relationship between dispersion observed on July 12, 1989 (red symbols) and the ones theoretically calculated by Model 1 (green lines).

gate along a magnetic field line by cutting across the equatorial plane over the time period from 19:13 to 19:42 UT, and they were detected in the northern hemisphere. After 19:42 UT, the lightning whistlers were detected in the southern hemisphere. In comparison, as shown in Fig. 9 when Akebono orbited in the further points from the source of lightning whistlers, the observed dispersion trend tends to deviate from the dispersion curve of $D_{1}$.

On the other hand, Fig. 10 shows the observed dispersion trend and the line of dispersion $D_{2}$ theoretically calculated by using Eq. (22). Also note that the scale for $D_{2}$ is different from the observed dispersion. As seen in Fig. 10, the dispersion $D_{2}$ tends to slightly deviate away from the observed dispersion trend. However, it shows a better model than the one shown in Fig. 9.

Same analyses were applied to the event on July 12, 1989. Figure 11 shows the observed dispersion trends and two lines for the dispersion $D_{1}$ that calculated theoretically

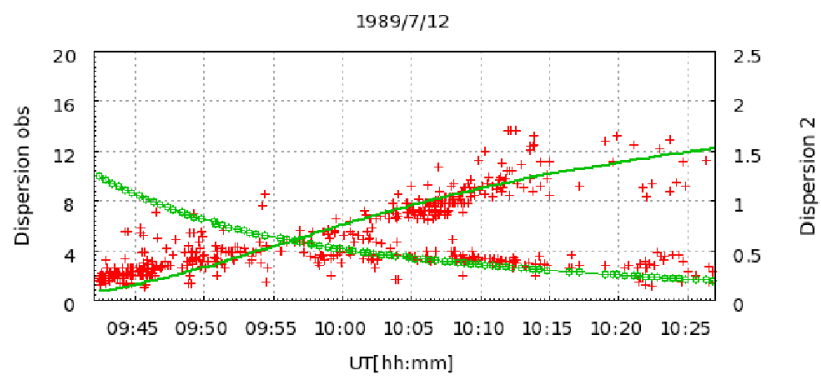

Fig. 12 Relationship between dispersion observed on July 12, 1989 (red symbols) and the ones theoretically calculated by Model 2 (green lines).

by using Eq. (20). The "bullet" line represents decreasing dispersion and the "smooth" line indicates increasing dispersion theoretically derived from Eq. (20). We can clearly observe that the bullet line appears like a curved line between 09:42 and 09:56 UT. During this time, the lightning whistlers originated from the southern hemisphere and were detected in the northern hemisphere by cutting across the equatorial plane. After 09:56 UT, the dispersion $D_{1}$ looks like a straight line. As represented in Fig. 11, when Akebono orbited at lower altitudes in the earlier time, the dispersion $D_{1}$ of the "bullet" line is not parallel with the observed dispersion trend.

The smooth line in Fig. 11 appears to be almost a straight line. As stated previously, sources of lightning whistlers that correspond to increasing dispersion originate from the northern hemisphere and the observed dispersion trend also appears to be almost straight. The dispersion $D_{1}$ of the "smooth" line is almost parallel with the observed dispersion trend.

Figure 12 shows the observed dispersion trends and the lines of dispersion $D_{2}$ theoretically calculated by using Eq. (22). Although the green lines have slight deviation from those of observed trends in the earlier times in the higher latitude region of northern hemisphere, they show a better model than the ones shown in Fig. 11.

As was shown in Figs. 9, 10, 11 and 12, we demonstrated that the observed dispersion trends are almost fit to the ones theoretically derived. As far as we examine these 3 dispersion trends, theoretical dispersion $D_{2}$ derived from Model 2 gives better agreement with these observations than the ones derived from Model 1. We should note that our analyses are rather simple that we just assume that the whistler mode wave propagates strictly along the geomagnetic field line with its wave normal parallel to the magnetic field line, and it is necessary to determine more precise propagation path using ray tracing technique. In addition there might be other solutions of the electron density profile to give agreement with the observation. However, the results of our research suggest that it is plausibly possible to determine electron density profile using observed dispersion trend of lightning whistler every trajectory of Akebono. 


\section{Conclusion}

In the present paper, we first showed that we can approximately derive the dispersion of lightning whistler and the electron density profile strongly affects the trend of dispersion in the plasmasphere. Second, we introduced two typical events observed by Akebono in which lightning whistlers were continuously observed along the satellite's trajectory. We demonstrated that the trends of dispersion of the lightning whistlers basically give good agreement with the ones theoretically derived. We also showed that the calculated dispersions using the electron density profile of Model 2 was better fit to the observation results. As shown previously, some deviations appeared because some parameters used when deriving the formula were assumed. In addition, it is also necessary to determine more precise propagation path using ray tracing technique and consider ionospheric contribution in deriving dispersion $D$. But our investigation proved that analyzing the dispersion trends of lightning whistler observed in the plasmasphere is a powerful method to estimate global electron density profile in the plasmasphere. As was described in the introduction, Akebono has continuously observed since 1989 and still in operation now, and enormous data of WBA waveform data is very valuable to study long term variation of electron density profile in the plasmasphere statistically.

\section{Acknowledgments}

We thank to our colleague Mr. Le Hoai Tam for his assistance in preparing the Akebono lightning whistler database.

\section{References}

[1] I. Kimura, K. Hashimoto, I. Nagano, T. Okada, M. Yamamoto, T. Yoshino, H. Matsumoto, M. Ejiri, and K. Hayashi, "VLF observations by the Akebono (EXOS-D) satellite," J. Geomag. Geoelectr., vol.42, pp.459-478, 1990.

[2] Y. Kasahara, A. Hirano, and Y. Takata, "Similar data retrieval from enormous datasets on ELF/VLF wave spectrum observed by Akebono," Data Science Journal, vol.8, pp.IGY66-IGY75, March 2010.

[3] J. Chum, F. Jirick, O. Santolik, M. Parrot, G. Diendorfer, and J. Fisr, "Assigning the causative lightning to the whistlers observed on satellites," Annales Geophysical, vol.24, pp.2921-2929, Nov. 2006.

[4] J. Oster, A.B. Collier, A.R.W. Hughes, L.G. Blomberg, and J. Lichtenberger, "Spatial correlation between lightning strikes and whistler observation and whistler observation from Tihany, Hungary," South African Journal of Science, vol.105, pp.234-237, May/June 2009.

[5] D.L. Carpenter, "Remote sensing of the magnetospheric plasma by means of whistler mode signals," Reviews of Geophysics, vol.26, no.3, pp.535-549, Aug. 1988.

[6] J. Crouchley, "A study of whistling atmospherics," Aust. J. Phys., vol.17, pp.88-105, Aug. 1964.

[7] J.D. Menietti and D.A. Gurnett, "Whistler propagation in jovian magnetoshere," Geophysical Research Letters, vol.7, no.1, pp.4952, Jan. 1980.

[8] G.J. Daniell, "Approximate dispersion formulae for whistler," J. Atmospheric Terrestrial Physics, vol.48, no.3, pp.267-270, Aug. 1985

[9] R.L. Smith and J.J. Angeram, "Magnetospheric properties deduced from OGO 1 observations of ducted and nonducted whistler,' J. Geophysical Research, vol.73, pp.1-20, Jan. 1968.

[10] O. Santolik, M. Parrot, U.S. Inan, D. Buresova, D.A. Gurnett, and J. Chum, "Propagation of unducted whistlers from their source lightning: A casestudy," J. Geophysical Reserach, vol.114, pp.1-11, March 2009.

[11] J. Lichtenberger, C. Ferencz, D. Hamar, P. Steinbach, C. Rodger M. Clilverd, and A. Collier, "Automatic retrieval of plasmaspheric electron densities: First results from automatic whistler detector and analyzer network," General Assembly and Scientific Symposium, 2011 XXXth URSI, Aug. 2011.

[12] Y. Goto, Y. Kasahara, and T. Sato, "Determination of plasmaspheric electron density profile by stochastic approach," Radio Science, vol.38, no.3, 1060, doi:10.1029/2002RS002603, June 2003.

[13] F. Akalin, D.A. Gurnett, T.F. Averkamp, A.M. Persoon, O. Santolik, W.S. Kurth, and G.B. Hospodarsky, "First whistler observed in the magnetosphere of Saturn," Geophysical Research Lett., vol.33, L20107, pp.1-5, 2006.

[14] R. Singh, A.K. Singh, and R.P. Singh, "Synchronized whistlers recorded at Varanasi," Pramana J. Physics, vol.60, no.6, pp.12731277, June 2003

[15] D.A. Gurnett, W.S. Kurth, I.H. Cairns, and L.J. Granroth, "Whistler in Neptune's magnetosphere: Evidence of atmospheric lightning," J. Geophysical Research, vol.95, no.A12, pp.20,967-20,976, Dec. 1990.

[16] B.C. Edgar, "The upper and lower frequency cutoff of magnetospherically reflected whistler," J. Geophysicical Research, vol.81, pp.205-211, 1976.

[17] J.G. Luhmann and L.M. Friesen, "A simple model magnetosphere," vol.84, pp.4405-4408, Aug. 1979.

[18] G.W. Prolss, Physics of the Earth's space environment an introduction, Springer, 2004.

[19] C.E. McIlwain, "Magnetic coordinates," Space Science Reviews, vol.5, no.5, pp.585-598, 1965.

[20] D.L. Carpenter, "Electron-density variations in the magnetosphere deduced from whisler data," J. Geophysical Research, vol.67, no.9, pp.3345-3360, Aug. 1962.

[21] D.L. Carpenter and R.L. Smith, "Whistler measurements of electron density in the magnetosphere," Reviews of Geophysics, vol.2, no.3, pp.415-441, March 1964.

[22] R.L. Smith, "The use of whistlers in the study of the outer ionosphere," Tech. Rep. 6, Radioscience Lab., Stanford Univ., Stanford, Calif., Oct. 1960

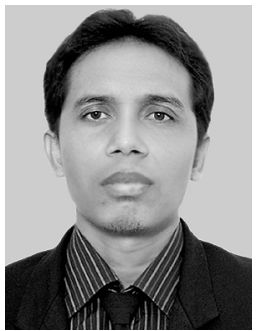

I Putu Agung Bayupati received the B.Sc degree in Electrical Engineering from Udayana University and M.Sc. degree in Information Technology from Bandung Institute of Technology in 2000 and 2006, respectively. Currently, he is a Ph.D. student at Graduate School of Natural Science and Technology, Kanazawa University, Japan and is studying intelligent signal processing for whistler detection. 


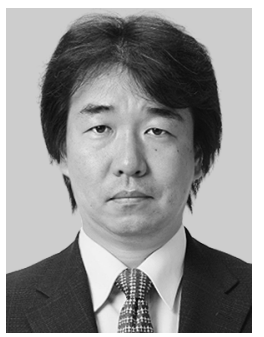

Yoshiya Kasahara received the B.E., M.E. and Ph.D. degrees in the field of electrical engineering from Kyoto University in 1989, 1991 and 1996, respectively. He had been a research associate of Kyoto University since 1992, and joined to Kanazawa University as an associate professor in Department of Information and Systems Engineering in 2002. He is currently a professor in Information Media Center, Kanazawa University. His research interests are in radio engineering and radio science, intelligent signal processing for measurements of plasma waves onboard spacecraft, theoretical studies on generation and propagation mechanism of waves in space plasma, and database system for space environment. He is a member of Society of Geomagnetism and Earth, Planetary and Space Sciences (SGEPSS), Information Processing Society of Japan (IPSJ) and American Geophysical Union (AGU).

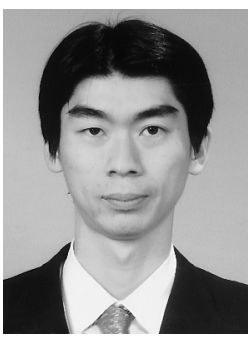

Yoshitaka Goto received the B.E. degree in electrical engineering, and M.I. (Master of Infomatics), and Ph.D. degrees in Communications and Computer Engineering from Kyoto University in 1998, 2000 and 2003, respectively. He joined to Kanazawa University as a research associate in 2003. Now he is an assistant professor at School of Electrical and Computer Engineering, Kanazawa University. He was a visiting researcher at the NASA Goddard Space Flight Center in 2007. His major research interests are in radio science and engineering; particularly signal processing technique for plasma waves observed by scientific satellites. He was awarded URSI Young Scientist Award in 2002. He is a member of the Society of Geomagnetism and Earth, Planetary and Space Sciences (SGEPSS). 